\title{
Multiple Mechanisms of Consciousness: The Neural Correlates of Emotional Awareness
}

\author{
Jayna M. Amting, ${ }^{1,2 \star}$ Steven G. Greening, ${ }^{2 \star}$ and Derek G. V. Mitchell ${ }^{1,2}$ \\ ${ }^{1}$ Department of Psychiatry and ${ }^{2}$ Department of Anatomy and Cell Biology, Schulich School of Medicine and Dentistry, The University of Western Ontario, \\ London, Ontario, Canada N6A 5A5
}

\begin{abstract}
Emotional stimuli, including facial expressions, are thought to gain rapid and privileged access to processing resources in the brain. Despite this access, we are conscious of only a fraction of the myriad of emotion-related cues we face everyday. It remains unclear, therefore, what the relationship is between activity in neural regions associated with emotional representation and the phenomenological experience of emotional awareness. We used functional magnetic resonance imaging and binocular rivalry to delineate the neural correlates of awareness of conflicting emotional expressions in humans. Behaviorally, fearful faces were significantly more likely to be perceived than disgusted or neutral faces. Functionally, increased activity was observed in regions associated with facial expression processing, including the amygdala and fusiform gyrus during emotional awareness. In contrast, awareness of neutral faces and suppression of fearful faces were associated with increased activity in dorsolateral prefrontal and inferior parietal cortices. The amygdala showed increased functional connectivity with ventral visual system regions during fear awareness and increased connectivity with perigenual prefrontal cortex (pgPFC; Brodmann's area 32/10) when fear was suppressed. Despite being prioritized for awareness, emotional items were associated with reduced activity in areas considered critical for consciousness. Contributions to consciousness from bottom-up and top-down neural regions may be additive, such that increased activity in specialized regions within the extended ventral visual system may reduce demands on a frontoparietal system important for awareness. The possibility is raised that interactions between pgPFC and the amygdala, previously implicated in extinction, may also influence whether or not an emotional stimulus is accessible to consciousness.
\end{abstract}

\section{Introduction}

The conscious perception of visual stimuli is thought to be an adaptation that complements reflexive unconscious representation, allowing for more sophisticated planning and action (Crick and Koch, 2003). Visual awareness is associated with increased cortical activity (Bar et al., 2001), particularly in category-selective "bottom-up" sensory areas within the ventral visual system (Tong et al., 1998; GrillSpector et al., 2000). Although increased stimulus representation is considered a prerequisite for conscious perception, it does not inevitably lead to awareness. Robust activity to unperceived stimuli has been observed throughout visual cortex (Leopold and Logothetis, 1996), fusiform gyrus (Moutoussis and Zeki, 2002), and motor cortex (Dehaene et al., 1998). Thus, awareness depends not only on activation in category-selective regions, but also on input from superordinate structures. Candidate regions for performing this "topdown" function include lateral prefrontal and inferior parietal cortices (Beck et al., 2001; Vuilleumier et al., 2001a). Indeed, a recent lesion study suggests a causal role for prefrontal cortex in allowing

\footnotetext{
Received Dec. 30, 2009; revised May 28, 2010; accepted June 8, 2010.

This research was supported by a grant to D.G.V.M. from the Natural Science and Engineering Research Council of Canada. We thank Dr Richard Neufeld for very helpful consultation on matters concerning the data analysis. We also thank Joy Williams for assistance with scanning, Karan Gupta for help in preparing the stimuli, and Betsy Schaefer for participant recruitment and screening.

*.M.A. and S.G.G. contributed equally to this study.

Correspondence should be addressed to Dr. Derek Mitchell, University Hospital, University of Western Ontario, 339 Windermere Road, London, Ontario, Canada N6A 5A5. E-mail: dmitch8@uwo.ca

DOI:10.1523/JNEUROSCI.6434-09.2010

Copyright $\odot 2010$ the authors $\quad 0270-6474 / 10 / 3010039-09 \$ 15.00 / 0$.
}

stimuli access to consciousness (Del Cul et al., 2009). Although the precise functional contribution of these neural regions remain unclear (Rees, 2007), frontoparietal areas may facilitate awareness by amplifying relevant sensory processes (Dehaene and Naccache, 2001) or biasing the content of awareness toward internal representations (Lumer et al., 1998).

Whereas the neural regions that make bottom-up contributions to visual awareness appear to be category selective (Lumer et al., 1998; Tong et al., 1998), top-down frontoparietal regions appear to factor in consciousness in a stimulus-independent or "generic" manner (Beck et al., 2001; Rees, 2007). However, the neural basis of visual awareness has been examined in only a limited range of stimuli. The possibility therefore remains that frontoparietal contributions to consciousness vary as a function of the properties of the stimuli subject to awareness, suppression, or both. Because of the special processing properties of emotions (Vuilleumier and Driver, 2007), comparing emotional versus neutral facial expressions is particularly promising for extending current models of consciousness. Studies of attention suggest that emotional stimuli receive special access to representation in subcortical areas (Vuilleumier et al., 2001b) even without normal awareness (Vuilleumier et al., 2002). However, it is unclear whether, in the absence of changes in stimulus properties or task demands, subcortical areas contribute to consciousness or alter the contribution of other structures implicated in awareness.

In the present functional magnetic resonance imaging (fMRI) study, we used binocular rivalry to test two opposing predictions about emotional consciousness. If top-down structures make a generic (emotion-independent) contribution to emotional 
awareness, then activity in bottom-up (ventral visual) but not top-down (frontoparietal) areas should vary as a function of the perceived facial expression. Alternatively, top-down and bottom-up contributions may be additive, such that enhanced contributions from specialized bottom-up structures place fewer demands on a generic top-down mechanism for awareness. Lastly, we tested the intriguing hypothesis that, given the role of perigenual prefrontal cortex [pgPFC; Brodmann's area (BA) 32/10] in emotion regulation (Ochsner and Gross, 2005) and extinction (Quirk and Gehlert, 2003), enhanced functional connectivity between the amygdala and pgPFC would be associated with the suppression of fear from awareness.

\section{Materials and Methods}

Subjects. Sixteen healthy human subjects (6 male and 10 female) with a mean age of 24.88 (range 20-32; SD 2.71) completed the study. All subjects granted informed consent, were in good health, and had no past history of psychiatric problems, neurological disease, or head injury as determined by screening and interview using the Structured Clinical Interview for DSM-IV (Diagnostic and Statistical Manual of Mental Disorders, 4th Edition). All subjects had normal or corrected to normal vision and were screened for color blindness using the Ishihara color blindness test. All subjects were right handed as determined by the Edinburgh handedness inventory. The study was approved by the Health Sciences Research Ethics Board at the University of Western Ontario, London, Ontario, Canada.

Stimuli. A binocular rivalry task was used to manipulate awareness of facial expressions.

Binocular rivalry occurs when discrepant images are presented to each eye. This manipulation creates perceptual rivalry, in which one image is perceived while the other is suppressed from awareness. Because the technique varies visual awareness while holding stimulus input and task demands constant, it is considered an effective means for exploring visual consciousness (Tong et al., 2006). Experimental conditions included competing displays of superimposed red and blue facial expressions (see Fig. 1). To induce binocular rivalry, participants wore glasses with a red filter over one eye and a blue filter over the other. The filter/eye pair was counterbalanced across subjects (i.e., right eye with red filter and left eye with blue filter or vice versa). Large numbers of unique individual stimuli were included to minimize habituation (Wright et al., 2001) and also served to reduce the potential influence of perceptual memory traces on subsequent awareness (cf. Pearson and Brascamp, 2008; Sterzer and Rees, 2008). Consequently, facial expressions were taken from 48 different actors (24 males) from the empirically validated NimStim Face Stimulus Set (Tottenham et al., 2002) and the Karolinska Directed Emotional Faces (Lundqvist et al., 1998). The facial images were cropped to remove the hair and neck to eliminate extraneous stereotypic features. Color filters were then applied in Adobe Photoshop to render sets of "red only" and "blue only" stimuli. Images were rendered semitransparent, standardized for luminosity and contrast, and superimposed on one another.

There were a total of six different conditions (three nonrivalrous conditions and three rivalrous conditions). The nonrivalrous conditions included the following: (1) a fearful facial expression paired with the same stimulus (one in red and one in blue); (2) a disgusted facial expression paired with the same stimulus; and (3) a neutral facial expression paired with the same stimulus. The rivalrous conditions included these: (1) a fearful facial expression paired with a neutral facial expression (a red fearful face paired with a blue neutral face and vice versa); (2) a disgusted facial expression paired with a neutral facial expression; and (3) a fearful facial expression paired with a disgusted facial expression. In each case, the two superimposed faces that formed a stimulus, whether nonrivalrous or rivalrous, were of the same actor. In each condition, the red-blue designation of each face was counterbalanced (i.e., for each actor, there was one trial involving a red fearful face paired with a blue neutral face and a second trial involving a blue fearful face paired with a red neutral face). Thus, for each actor (48), there were 12 presentations ( 6 stimuli presented twice to balance color). Altogether, there were 96 trials for each condition for a total of 576 trials divided equally into 6 runs.

Task design. Each trial began with a fixation point $(500 \mathrm{~ms})$, followed by the image of two superimposed facial expressions (rivalrous or nonrivalrous; $500 \mathrm{~ms}$ ) and then a screen listing the four potential emotional perception choices ( $2250 \mathrm{~ms}$ ). During binocular rivalry, perceptual dominance spontaneously alternates every few seconds between one image and the other (Tong et al., 2006). As with other recent fMRI studies with similar considerations (e.g., Williams et al., 2004), a short presentation time for each stimulus was chosen specifically to help insure that participants would experience a single unchanging percept on each trial. While undergoing fMRI, participants indicated whether the facial expression presented was neutral, fearful, disgusted, or blended (if they could not distinguish one single emotion) via button press at the end of every trial. 
Disgusted faces were chosen to complement the fearful and neutral stimuli for two main reasons. First, like fearful stimuli, disgusted faces involve complex pattern information and luminance contrast edges within the expressive features; i.e., for both fearful and disgusted faces, the mouth, nose, eye, and eyebrows all change position relative to their location on a neutral expression (Ekman and Friesen, 1975). Because both disgusted and fearful faces involve this complex pattern information, it is less likely that participants would make rule-based (e.g., if eye brows deviate from center respond "fear") rather than emotional discriminations. This is particularly true in the context of piecemeal rivalry, where if only fearful and neutral expressions were present, participants may be more likely to form a response bias to label blended percepts as "fearful" by default. Second, relative to fearful faces, disgusted expressions are thought to activate a partially dissociable network of neural regions (Calder et al., 2001) and receive less prioritized access to processing resources (Anderson et al., 2003). There were 96 trials in each condition for a total of 576 trials divided equally into 6 runs. Within each run, the six different stimulus conditions were intermixed, presented in random order, and balanced. The runs themselves were presented in pseudorandom order. The stimuli were projected through a data projector onto a screen that could be viewed by the subject via a mirror positioned above the head-coil in the MRI scanner. Before entering the scanner, subjects performed a 24 trial training version that included samples of each condition that were not used in the experiment. The task was programmed using E-Prime software (Schneider et al., 2002).

Behavioral data analysis. Because of the possibility that perception of an expression was driven in part by emotion recognition difficulty (e.g., the expressions may differ intrinsically in the ease with which they are identified), we controlled for the ability to correctly identify fearful (F), neutral $(\mathrm{N})$, and disgusted (D) faces in nonrivalrous conditions. Thus, a "fear awareness score" was derived by dividing the number of times fear was present and perceived in rivalrous conditions (fear paired with neutral or disgusted faces) by the number of correct identifications of fear made in nonrivalrous conditions $[(\mathrm{FNF}+\mathrm{FDF}) / \mathrm{FF}]$. A disgust awareness score was derived by dividing the number of times disgust was present and perceived in rivalrous conditions (disgust paired with neutral or fearful faces) divided by the number of correct identifications of disgust made in nonrivalrous conditions $[(\mathrm{DND}+\mathrm{FDD}) / \mathrm{DD}]$. A neutral awareness score was derived in the same fashion [(DNN + FNN)/ $\mathrm{NN}$ ]. A repeated-measures ANOVA was used to determine whether the three awareness scores differed significantly. In addition, to examine the relative likelihood of unresolved rivalry across conditions, a second ANOVA was conducted examining the number of "blended" responses across the three rivalrous conditions (fear paired with disgust; fear paired with neutral; disgust paired with neutral). Follow-up paired $t$ tests were conducted to delineate the nature of significant effects.

MRI data acquisition. Subjects were scanned during task performance using a 3T Siemens scanner with a 32 channel head coil. fMRI images were taken with a $\mathrm{T} 2{ }^{\star}$-gradient echo-planar imaging sequence [repetition time $=3000 \mathrm{~ms}$; echo time $=30 \mathrm{~ms} ; 120 \times 120$ matrix; field of view $(\mathrm{FOV})=24 \mathrm{~cm}]$. Complete brain coverage was obtained with 45 ascending interleaved slices of $2 \times 2 \mathrm{~mm}$ in plane with a slice thickness of 2.5 $\mathrm{mm}$, which formed voxels of $2 \times 2 \times 2.5 \mathrm{~mm}$. A high-resolution, T1weighted, anatomical scan was obtained covering the whole brain (repetition time $=2300 \mathrm{~ms}$, echo time $=4.25 \mathrm{~ms} ; \mathrm{FOV}=25.6 \mathrm{~cm} ; 192$ axial slices; voxel size $=1 \mathrm{~mm}$ isovoxels; $256 \times 256$ matrix).

$f M R I$ analysis. Individual and group analyses were conducted using Analysis of Functional NeuroImages software (AFNI) (Cox, 1996). The first four "dummy" volumes of each of the six runs were discarded to insure that magnetization equilibrium was reached. Motion correction was performed by registering all volumes to the first functional volume acquired following the anatomical scan. The dataset for each subject was spatially smoothed (using an isotropic $4 \mathrm{~mm}$ Gaussian kernel) and the time series data were normalized by dividing the signal intensity of a voxel at each time point by the mean signal intensity of that voxel for each run and multiplying the result by 100 . The resultant regression coefficients represented the percentage signal change from the mean activity. Regressors for each of the 12 experimental conditions were created using a fixed duration epoch design by convolving the stimulus events of inter-

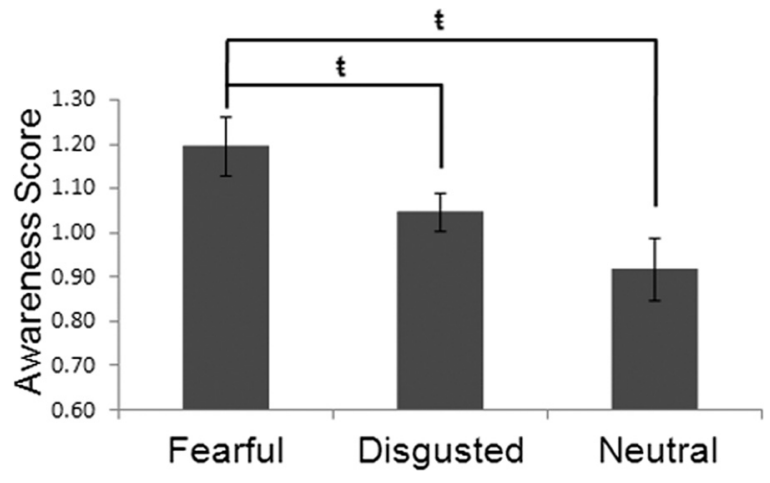

Figure 2. Fear is prioritized for awareness. Behavioral results demonstrated that participants were significantly more likely to be conscious of fearful facial expressions than both disgusted $\left(t_{(15)}=\right.$ $2.26, p=0.039)$ and neutral $\left(t_{(15)}=2.83, p=0.013\right)$ expressions when paired with a competing facial expression. Error bars depict the SEM between subjects $(\xi=p<0.05)$.

Table 1. Contrast tests for perceived fear and disgust

\begin{tabular}{|c|c|c|c|c|c|c|}
\hline Anatomical location & $L / R$ & $\mathrm{BA}$ & $x$ & $y$ & $z$ & $t$ value \\
\hline \multicolumn{7}{|c|}{ Perceived fear vs unperceived fear contrast } \\
\hline \multicolumn{7}{|c|}{ Perceived fear $>$ unperceived fear } \\
\hline Amygdala* & $\mathrm{R}$ & & 16 & -8 & -12 & 4.29 \\
\hline \multicolumn{7}{|c|}{ Unperceived fear $>$ perceived fear } \\
\hline Perigenual PFC & $\mathrm{L}$ & 24 & -3 & 33 & 7 & 6.79 \\
\hline \multicolumn{7}{|c|}{ Perceived disgust vs unperceived disgust contrast } \\
\hline \multicolumn{7}{|c|}{ Perceived disgust $>$ unperceived disgust } \\
\hline Fusiform gyrus* & $\mathrm{L}$ & 37 & -35 & 48 & -22 & 5.78 \\
\hline \multicolumn{7}{|c|}{ Unperceived disgust $>$ perceived disgust } \\
\hline Inferior parietal cortex & $\mathrm{R}$ & 40 & 62 & -40 & 38 & 6.90 \\
\hline \multicolumn{7}{|c|}{$\begin{array}{l}\text { Emotion suppression with neutral awareness }> \\
\text { neutral neutral }\end{array}$} \\
\hline Middle frontal gyrus & $\mathrm{R}$ & $9 / 44$ & 48 & 9 & 39 & 8.13 \\
\hline Middle frontal gyrus \% & $\mathrm{L}$ & $9 / 45$ & -40 & 28 & 32 & 7.71 \\
\hline Inferior frontal gyrus & $\mathrm{R}$ & 44 & 50 & 12 & 20 & 6.31 \\
\hline Insula & $\mathrm{L}$ & 13 & -32 & 18 & 6 & 6.64 \\
\hline Insula & $\mathrm{R}$ & 13 & 30 & 20 & 4 & 6.11 \\
\hline Precuneus & $\mathrm{R}$ & 7 & 6 & 67 & 42 & 6.60 \\
\hline
\end{tabular}

Significant neural regions revealed by the perceived fear versus unperceived fear, perceived disgust versus unperceived disgust, and neutral awareness with emotional suppression contrast tests. Displayed in the table are hemispheric location (L, left; R, right), Brodmann's area (BA), Montreal Neurological Institute coordinates at the center of mass $(x, y, z)$, and maximum activity ( $F$ value) for each significant cluster. $p<0.001 ; p<0.05$, corrected; ${ }^{*} p<0.01 ; \%$ uncorrected.

est (i.e., $500 \mathrm{~ms}$ of superimposed facial expression presentation) with a gamma-variate basis function to account for the slow hemodynamic response. The BOLD response was fitted to each of the 13 regressors to perform linear regression modeling. The rivalrous disgust-neutral condition (DN) yielded three possible regressors; participants perceived either disgusted (DND), neutral (DNN), or blended (DNB) faces. The rivalrous fearful-neutral condition (FN) also yielded three regressors; participants perceived either fearful (FNF), neutral (FNN), or blended (FNB) faces. The rivalrous fear-disgust condition (FD) yielded three possible regressors; participants perceived either fearful (FDF), disgusted (FDD), or blended (FDB) faces. There were also three regressors generated from nonrivalrous pairings of fearful (FF), disgusted (DD), and neutral (NN) faces. Errors were defined as those trials in which participants indicated awareness for an emotion that was not present. Such trials, along with responses made outside of a $2250 \mathrm{~ms}$ time period, were modeled separately as regressors of no interest. To account for voxelwise correlated drifting, a baseline plus linear drift and quadratic trend were modeled to the time series of each voxel. This produced a $\beta$ coefficient and $t$ value for each voxel and regressor. To perform the group analyses, each individual's data were transformed into the standard space of Talairach and Tournoux. This individual subject analysis was followed by the group analyses described below. 
Group analysis. Two contrast tests were performed to examine neural activity associated with the awareness of fearful and disgusted facial expressions. A perceived fear versus unperceived fear contrast compared the mean blood oxygenation level-dependent (BOLD) response associated with all rivalrous trials in which fear was present and perceived (FNF and FDF) with rivalrous trials in which fear was present but not perceived (FNN and FDD). A contrast examining perceived fear versus unperceived fear was defined by the following equation: $[(\mathrm{FNF}+\mathrm{FDF}) / 2]-[(\mathrm{FNN}+$ FDD)/2]. The perceived disgust versus unperceived disgust contrast compared the mean BOLD response associated with all rivalrous trials in which disgust was present and perceived (DND and FDD) with all rivalrous trials in which disgust was present but not perceived (DNN and FDF). The contrast examining perceived disgust versus unperceived disgust was defined by the following equation: [(DND + $\mathrm{FDD}) / 2]-[(\mathrm{DNN}+\mathrm{FDF}) / 2]$.

Following the suggestion of an anonymous reviewer, we performed a third contrast to dissociate brain activity specifically related to the awareness of a neutral stimulus while suppressing an emotional stimulus. At the same time, the contrast allowed us to control for activity associated with the perception of a neutral stimulus in the absence of emotional rivalry. This contrast compared the BOLD response in all conditions where an emotional face was present but unperceived (i.e., a fearful or disgusted face was suppressed) and a neutral face was perceived (FNN and DNN) relative to trials in which only neutral faces were present and perceived (NN). This contrast of perceived neutral with emotion suppression versus neutral only was defined by the following equation: $[(\mathrm{FNN}+\mathrm{DNN}) / 2]-\mathrm{NN}$.

In addition, a 3 (perceived emotion: fearful, disgusted, or neutral facial expressions) $\times 3$ (unperceived emotion: fearful, disgusted, or neutral facial expressions) ANOVA was conducted on the regression coefficients derived from the fMRI analysis (described above). For the main effect of perceived emotion, main effect of unperceived emotion, and interaction between the perceived and unperceived emotions, regions significantly active at a threshold of $p<0.001$ were examined. For a priori regions of interest (ROI) including the amygdala, fusiform gyrus, and anterior insula, a more liberal threshold was used $(p<0.01)$ and reported where significant. To correct for multiple comparisons, a spatial clustering operation was performed at a threshold of $p<0.001$ ( $p<0.05$ corrected) using AlphaSim with 1000 Monte Carlo simulations taking into account the entire echoplanar image matrix. For significantly active clusters, follow-up analyses using paired $t$ tests were performed to delineate the nature of the main effects and interaction.

Context-dependent connectivity analysis (psychophysiological interaction). One of the objectives of the current study was to identify neural regions that are important for selecting between conflicting emotional stimuli for awareness. Given the role of the amygdala in processing emotional facial expressions (Adolphs et al., 1994; LeDoux, 1996), we predicted that areas involved in the conscious perception of fear would show enhanced connectivity with the amygdala during trials of perceived relative to unperceived fear. Context-dependent changes in functional connectivity were assessed using a psychophysiological interaction model (Context-Dependent Correlation Analysis by Gang Chen, National Institute of Mental Health, Bethesda, MD; http://afni.nimh.nih.gov/ sscc/gangc/CD-CorrAna.html) that identified brain regions showing significantly altered functional connectivity (correlated activity) with the right amygdala during trials in which fear was perceived relative to the when fear was present but unperceived. Each individual's BOLD signal time series was converted into standardized Talairach space according to their anatomical dataset. The right amygdala functional mask (SEED region) was then separately paired with each individual's dataset, the time series was extracted, and the trend was removed from the resulting time series. The psychophysiological variable was created as a product of a deconvolved extracted time series of the seed region and a vector coded for the desired condition ( 1 for the perceived fear condition, -1 for conditions when fear was unperceived, 0 for all other conditions). The variable created for the incongruent and congruent conditions were paired to the detrended SEED time series. To control for global drifting, the average signal across the whole brain (global signal) was used as a covariate in the correlation analysis. A voxelwise correlation analysis was conducted between each individual voxel's time series and that of the specified SEED region. The proportion of the variation that could be attributed to the correlation with the SEED was determined by squaring the resulting correlation coefficient. The correlation coefficients were converted to a Gaussian variable using a Fisher transformation formula to reduce the skew and normalize the distribution. To identify regions that showed a significant alteration in correlated activity with right amygdala across perceived and unperceived conditions, a $t$ test was performed on the transformed correlation coefficients.

\section{Results}

Behavioral data

A repeated-measures ANOVA examining the response awareness scores (i.e., scores designed to control for individual differences 
Table 2. Main effect of perceived emotion

\begin{tabular}{|c|c|c|c|c|c|c|}
\hline Anatomical location & $\mathrm{L} / \mathrm{R}$ & BA & $x$ & $y$ & $z$ & Fvalue \\
\hline \multicolumn{7}{|c|}{$\begin{array}{l}\text { Perceived fearful and disgusted faces }> \\
\text { neutral faces }\end{array}$} \\
\hline Inferior occipital cortex ${ }^{\beta}$ & $\mathrm{L}$ & 18 & -34 & -90 & -10 & 22.87 \\
\hline Fusiform gyrus $^{\beta}$ & $L$ & 37 & -36 & -46 & -18 & 16.99 \\
\hline Inferior frontal gyrus & $\mathrm{L}$ & $44 / 45$ & -44 & 15 & 22 & 30.05 \\
\hline Declive & $\mathrm{R}$ & 9 & 10 & -73 & -31 & 17.49 \\
\hline \multicolumn{7}{|c|}{$\begin{array}{l}\text { Perceived disgusted faces }>\text { fearful and } \\
\text { neutral faces }\end{array}$} \\
\hline Postcentral gyrus & $\mathrm{L}$ & 3 & -49 & -22 & 49 & 19.76 \\
\hline Cuneus & $\mathrm{L}$ & 18 & -13 & -92 & 18 & 13.62 \\
\hline \multicolumn{7}{|c|}{$\begin{array}{l}\text { Perceived neutral faces }>\text { perceived fearful } \\
\text { and disgusted faces }\end{array}$} \\
\hline Middle frontal gyrus & $\mathrm{L}$ & 9 & -35 & 29 & 40 & 32.60 \\
\hline Middle frontal gyrus & $\mathrm{R}$ & 8 & 26 & 18 & 50 & 22.21 \\
\hline Middle frontal gyrus & $\mathrm{L}$ & 8 & -22 & 28 & 51 & 19.46 \\
\hline Perigenual PFC & $\mathrm{L}$ & 32 & -6 & 36 & 20 & 17.37 \\
\hline Perigenual PFC & $L / R$ & 32 & 0 & 40 & 3 & 15.40 \\
\hline Posterior cingulate gyrus & $L / R$ & 31 & 0 & -39 & 39 & 38.23 \\
\hline Inferior parietal cortex & $\mathrm{R}$ & 40 & 52 & -53 & 32 & 41.97 \\
\hline Inferior parietal cortex* & $\mathrm{L}$ & 40 & -58 & -42 & 37 & 13.62 \\
\hline Superior parietal cortex & $\mathrm{L}$ & 7 & -19 & -58 & 66 & 15.50 \\
\hline Superior occipital cortex & $\mathrm{L}$ & 19 & -40 & -79 & 27 & 20.14 \\
\hline Middle temporal gyrus & $\mathrm{R}$ & 21 & 59 & -26 & -19 & 20.16 \\
\hline Middle temporal gyrus ${ }^{\alpha}$ & $\mathrm{R}$ & 21 & 60 & -43 & -3 & 15.12 \\
\hline Middle temporal gyrus & $\mathrm{L}$ & 21 & -62 & -26 & -16 & 19.28 \\
\hline Superior temporal gyrus & $\mathrm{L}$ & 22 & -55 & -20 & 9 & 12.38 \\
\hline Lentiform nucleus ${ }^{\alpha}$ & $\mathrm{R}$ & & 29 & -18 & 8 & 33.22 \\
\hline \multicolumn{7}{|c|}{$\begin{array}{l}\text { Perceived neutral and disgusted faces }> \\
\text { fearful faces }\end{array}$} \\
\hline Lingual gyrus & $L / R$ & 18 & 0 & -73 & 2 & 23.64 \\
\hline Cuneus & $\mathrm{L}$ & 18 & -10 & -73 & 15 & 13.05 \\
\hline
\end{tabular}

Areas showing a significant main effect of perceived emotion following the perceived emotion $\times$ unperceived emotion $\left(3 \times 3\right.$ ANOVA) whole-brain analysis; $p<0.05$, corrected; ${ }^{\beta}$ perceived disgusted faces $>$ fearful faces $>$ neutral faces; ${ }^{*}$ perceived neutral faces $>$ fearful faces $>$ disgusted faces; ${ }^{\alpha}$ perceived neutral faces $>$ disgusted faces $>$ fearful faces; $L$, left; $R$, right.

in emotion recognition) was significant $\left(F_{(2,30)}=3.83, p<0.05\right)$. Follow-up paired $t$ tests revealed that, when present, fearful faces were consciously perceived $[(\mathrm{FNF}+\mathrm{FDF}) / \mathrm{FF} ; M=1.20, \mathrm{SD}=$ 0.27 significantly more than disgusted [(DND + FDD)/DD; $\left.M=1.05, \mathrm{SD}=0.17 ; t_{(15)}=2.26, p<0.05\right]$ and neutral faces $\left[(\mathrm{DNN}+\mathrm{FNN}) / \mathrm{NN} ; M=0.92, S D=0.28 ; t_{(15)}=2.83, p=\right.$ $0.01]$. The results of this analysis suggest that when multiple facial expressions are present, even emotional ones, fearful faces are most likely to reach awareness (Fig. 2). The second ANOVA revealed that the number of blended responses across the three rivalrous conditions did not differ significantly $\left(F_{(2,30)}=1.76\right.$, $p>0.18)$ : mean blended responses to fearful faces paired with neutral faces was $6.94(\mathrm{SD}=9.54)$; mean blended responses to fearful faces paired with disgusted faces was $10.75(\mathrm{SD}=11.44)$; mean blended responses to disgusted faces paired with neutral faces was $11.25(\mathrm{SD}=14.19)$.

\section{fMRI results}

\section{Contrast tests}

Perceived fearful faces versus unperceived fearful faces. The perceived fearful faces versus unperceived fearful faces contrast (Table 1) revealed significantly greater activity in the amygdala $(p<0.01)$ (Fig. $3 a$ ) when participants were aware of fearful faces. When participants were unaware of a fearful face being present (i.e., fear suppression), there was greater activity in the left pgPFC $(p<0.001)$ (Fig. 3b).

Perceived disgusted faces versus unperceived disgusted faces. The contrast of perceived disgusted faces versus unperceived disgusted faces (Table 1) revealed significantly greater activation in fusiform gyrus when a disgusted face was perceived $(p<0.01)$ (Fig. $3 c$ ). When a disgusted face was present but unperceived, there was greater activation in right inferior parietal cortex (BA 40; $p<0.001$ ).

Perceived neutral with emotion suppression versus neutral only. We conducted a contrast targeting neural regions specifically related to the suppression of an emotional percept in favor of a neutral one. At the same time, we controlled for activity associated with neutral stimulus perception in the absence of emotional rivalry. This contrast identified significantly greater activation in bilateral middle frontal gyrus $(p<0.001)$ (see Fig. 5), inferior frontal gyrus, bilateral insula, and precuneus when emotional faces were being suppressed (Table 1). There were no clusters with significantly greater activity in the neutral only condition compared with the perceived neutral with emotion suppression condition.

\section{ANOVA}

Main effect of perceived emotion

Areas showing a significant main effect of perceived emotion include widespread regions of prefrontal cortex, parietal cortex, and the dorsal and ventral visual system $(p<0.001)$. Pairwise comparisons were performed to delineate the nature of the main effects. The delineation revealed three primary patterns of activity: (1) areas showing significantly greater activity for perceived fearful and disgusted faces relative to neutral faces; (2) areas showing greater activity to perceived disgusted faces relative to perceived neutral and fearful faces; (3) areas showing greater activity to perceived neutral faces relative to perceived fearful and disgusted faces. These regions and their distinct pattern of results are detailed in Table 2.

Of particular interest, left inferior occipital cortex (BA 18) (Fig. 3d) and left fusiform gyrus (BA 37) (Fig. 3e) showed significantly greater activity for fearful and disgusted faces relative to neutral faces and greater activity to disgusted relative to fearful faces (disgusted $>$ fearful $>$ neutral; $p<0.05$ ) (Table 2). Significantly greater activity for perceived fearful and disgusted expressions relative to perceived neutral expressions (fearful and disgusted $>$ neutral; $p<0.01$ ) was also observed in left inferior frontal gyrus (BA 44/45).

A number of regions showed a significantly greater activity for perceived neutral faces regardless of the unperceived stimulus (neutral $>$ disgusted and fearful), including bilateral pgPFC (BA 32; left, $p<0.01$; right, $p<0.001$ ) (Fig. 4), right posterior cingulate cortex (BA 31; $p<0.001$ ), bilateral middle frontal gyrus (BA $8 / 9 ; p<0.001$ ) (Fig. $5 a, b$ ), bilateral middle temporal gyrus (BA $21 ; p<0.001$ ), left superior temporal gyrus (BA 22; $p<0.001$ ), bilateral inferior parietal cortex (BA 40; $p<0.001$ ) (Fig. $5 c$, left; Fig. $5 d$, right), left superior parietal cortex (BA 7; $p<0.001)$, and left superior occipital cortex (BA19; $p<0.001$ ) (Table 2). With the exception of left inferior parietal cortex, all regions showed no significant differences in activity for perceived fearful faces relative to perceived disgusted faces. Left inferior parietal cortex (BA 40) showed significantly greater activity for neutral relative to both fearful and disgusted faces and greater activity to fearful than disgusted faces (neutral $>$ fearful $>$ disgusted; $p<0.05$ ).

\section{Main effect of unperceived emotion}

There were no significant clusters observed for the main effect of unperceived emotion $(p<0.001)$.

Perceived emotion $\times$ unperceived emotion interaction

There were no significant clusters observed for the perceived emotion $\times$ unperceived emotion interaction $(p<0.001)$. 
Context-dependent connectivity analysis Table 3 and Figure 6 show the results of the functional connectivity analysis. Superior temporal cortex (BA 36), middle temporal cortex (BA 38), and superior parietal cortex (BA 7) all showed enhanced functional connectivity with the right amygdala during trials of perceived fear relative to unperceived fear $(p<0.01)$. Conversely, the pgPFC (BA 32), superior frontal gyrus (BA 6), middle frontal gyrus (BA 6), posterior cingulate cortex (BA 13), middle temporal cortex (BA 21), and superior temporal cortex (BA 41) all showed enhanced connectivity with the right amygdala during trials of unperceived fear relative to perceived fear $(p<0.01)$.

\section{Discussion}

In the current study, binocular rivalry was used to determine whether activity in top-down neural regions implicated in visual awareness or emotion regulation vary as a function of consciousness. We reasoned that if top-down contributions to consciousness functioned in a stimulus-independent fashion, then activity in bottom-up (ventral visual system) but not top-down (frontoparietal) structures should vary as a function of the perceived emotion. When present, fearful faces were significantly more likely to be perceived than disgusted or neutral faces. Despite gaining preferred access to consciousness, the perception of fearful relative to neutral faces was associated with less activity in top-down areas considered critical for visual awareness. Enhanced activity in the amygdala and ventral visual stream was associated with fear awareness. Whereas fear perception was associated with enhanced activity in the amygdala, increased pgPFC activity was observed when fearful faces were present but unperceived. Similarly, whereas awareness of fear was associated with increased functional coupling between amygdala and temporal cortices, trials featuring suppressed fearful faces featured greater connectivity between amygdala and pgPFC.

A distinction should be made between stimulus representation, the focus of studies of attention, and visual awareness, which is targeted by the current study. Attention enhances the neural representation of target stimuli (Desimone and Duncan, 1995); it is considered a critical precursor for awareness by some (Driver and Mattingley, 1998; Dehaene et al., 2006) and a potential confound by others (Tse et al., 2005). Although the specific relationship between attention and consciousness remains unclear (Rees, 2007), it is evident that enhanced representation is not sufficient to produce
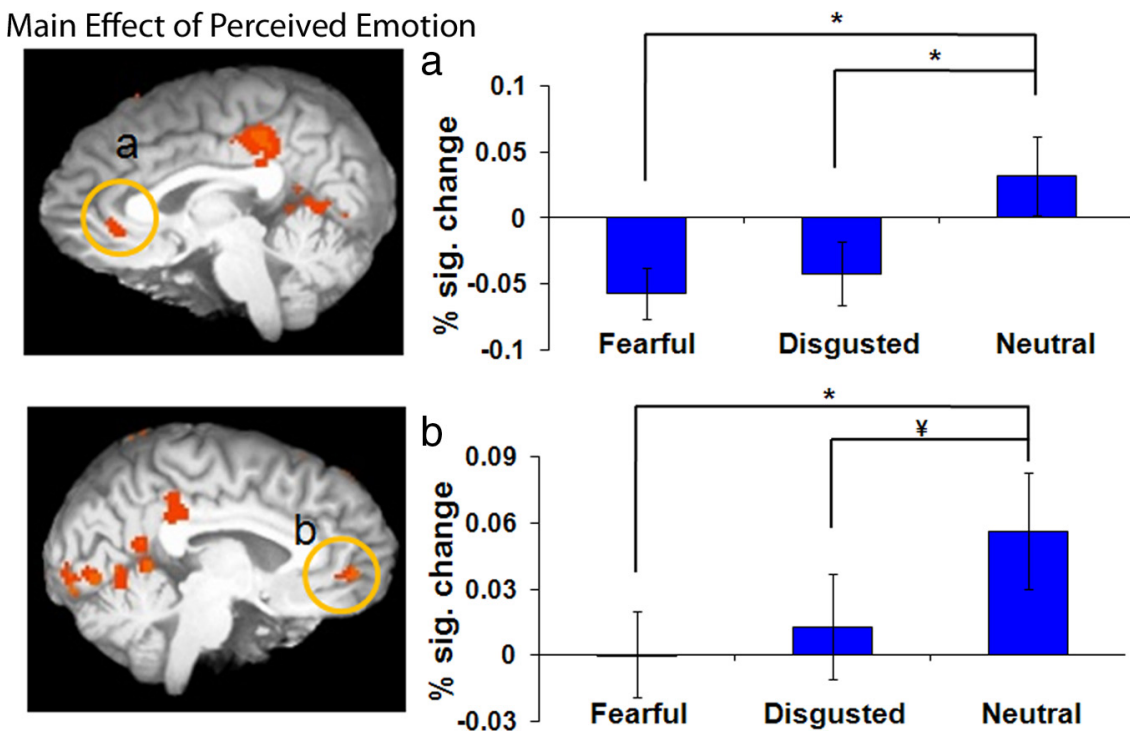

Figure 4. Perigenual prefrontal cortex activity associated with awareness of neutral relative to emotional stimuli. $\boldsymbol{a}, \boldsymbol{b}$, Images depicting the main effect of perceived emotion revealed greater activation in the right $(\boldsymbol{a})$ and left $(\boldsymbol{b})$ perigenual prefrontal cortex when a neutral expression was perceived relative to when either a fearful or disgusted expression was perceived $\left({ }^{*} p<0.001,{ }^{*} p<0.01\right)$. The $y$-axis depicts percentage signal change (\% sig. change) in BOLD response; error bars depict the SEM between subjects.
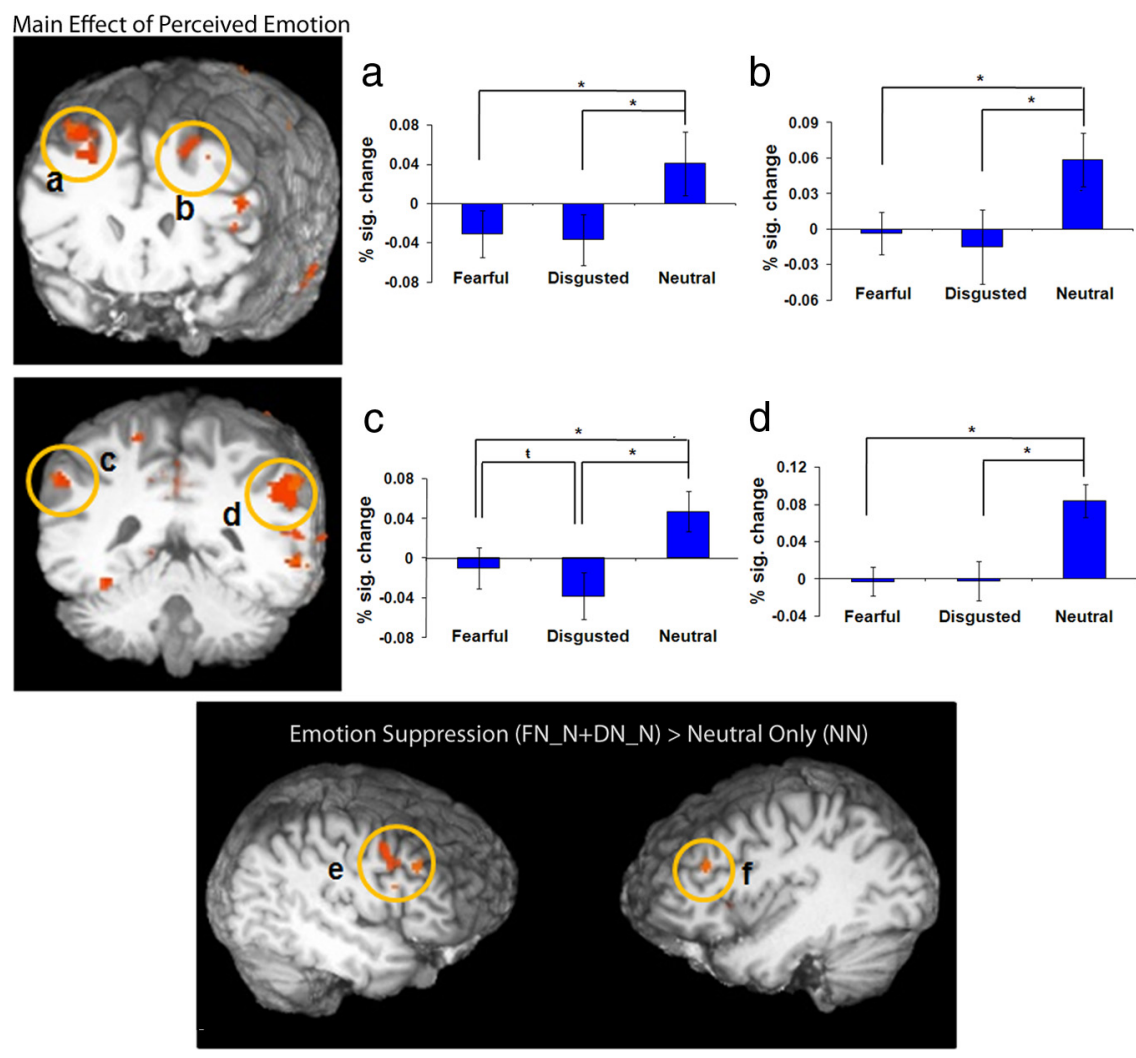

Figure 5. Enhanced activity in a frontoparietal network associated with awareness of neutral relative to emotional stimuli. $\boldsymbol{a}-\boldsymbol{d}$, Images depicting the main effect of perceived emotion revealed greater activation in the right $(\boldsymbol{a})$ and left $(\boldsymbol{b})$ middle frontal gyrus and the left $(\boldsymbol{c})$ and right $(\boldsymbol{d})$ inferior parietal cortices when a neutral relative to fearful or disgusted expression was perceived $\left({ }^{*} p<0.001,{ }^{*} p<0.05\right) . \boldsymbol{e}, \boldsymbol{f}$, The results of the contrast of emotion suppression [FN_N + DN_N] $>$ neutral only condition (NN) revealed greater activation in the right $(\boldsymbol{e})$ middle frontal gyrus and the left $(\boldsymbol{f})$ middle frontal gyrus during emotion suppression. The $y$-axis depicts percentage signal change in BOLD response; error bars depict the SEM between subjects.

awareness (Leopold and Logothetis, 1996). We controlled for stimulus duration, location, and task demands across conditions to target processes related to emotional awareness. Nevertheless, awareness depended on correctly categorizing a facial expression 
Table 3. Areas revealed by the functional connectivity analysis showing significantly enhanced or reduced functional connectivity with the right amygdala during fear awareness and fear suppression

\begin{tabular}{|c|c|c|c|c|c|c|}
\hline Anatomical location & $L / R$ & $B A$ & $x$ & $y$ & $z$ & $t$ value \\
\hline \multicolumn{7}{|c|}{$\begin{array}{l}\text { Regions showing greater amygdala connectivity } \\
\text { during perceived relative to unperceived fear }\end{array}$} \\
\hline Superior parietal cortex & $\mathrm{R}$ & 7 & 25 & -70 & 45 & 5.14 \\
\hline Middle temporal cortex & $\mathrm{R}$ & 38 & 42 & 7 & -13 & 5.52 \\
\hline Superior temporal cortex & $\mathrm{R}$ & 36 & 40 & -32 & -7 & 5.39 \\
\hline \multicolumn{7}{|c|}{$\begin{array}{l}\text { Regions showing greater amygdala connectivity } \\
\text { during unperceived relative to perceived fear }\end{array}$} \\
\hline Superior frontal gyrus & $L / R$ & 6 & 1 & 8 & 70 & 5.48 \\
\hline Middle frontal gyrus & $\mathrm{L}$ & 6 & -29 & 6 & 66 & 4.35 \\
\hline Perigenual PFC & $\mathrm{L}$ & 32 & -4 & 54 & 8 & 6.42 \\
\hline Posterior cingulate cortex & $\mathrm{L}$ & 31 & -8 & -69 & 18 & 4.51 \\
\hline Middle temporal cortex & $\mathrm{L}$ & 21 & -64 & -12 & -3 & 6.56 \\
\hline Superior temporal cortex & $\mathrm{L}$ & 41 & -62 & -28 & 12 & 4.57 \\
\hline
\end{tabular}

$p<0.01 ; L$, left; $R$, right.

more likely to be perceived than other expressions, and this awareness was associated with enhanced amygdala activity and increased functional coupling between amygdala and ventral visual system. Other evidence supports this finding. Subliminal emotional presentations modulate awareness for other stimuli (de Gelder et al., 2005); furthermore, subcortical influences likely contribute to residual emotion discrimination ability in affective blindsight (de Gelder et al., 1999) and rapid emotional contagion (Tamietto et al., 2009) for unseen stimuli in cortically blind patients. Furthermore, using signal detection theory, Pessoa et al. (2006) revealed greater amygdala activity to seen versus unseen fearful faces.

Although prior work has focused primarily on the extent to which awareness is required for emotional stimuli to activate the amygdala (Whalen et al., 1998; Pessoa et al., 2006), an alternate perspective is that amygdala activity influences the threshold for consciousness (Anderson and Phelps, 2001; Dehaene et al., 2006). Accordingly, the amygdala may not only detect innate biologically and socially relevant informa-
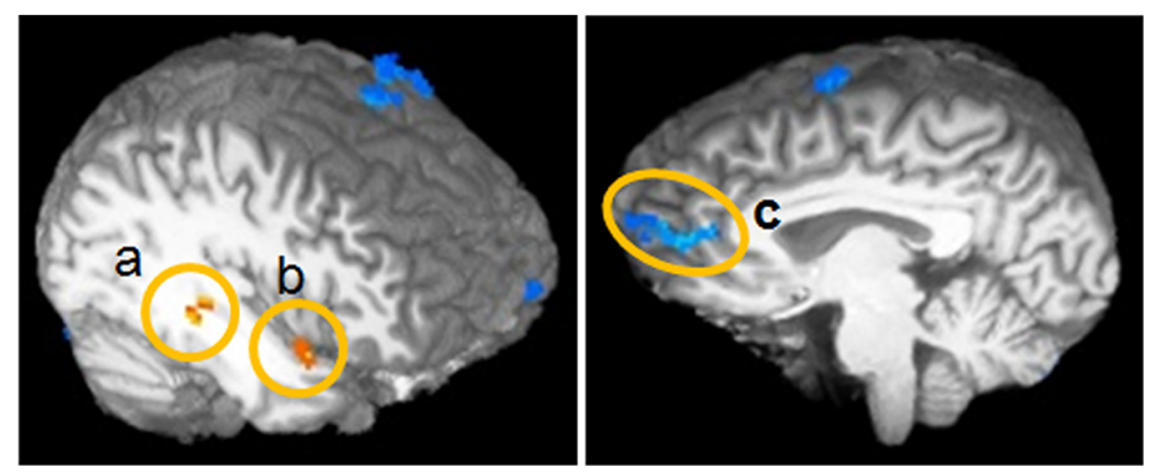
tion (Sergerie et al., 2008) but also facilitate awareness of such stimuli by interacting with phylogenetically newer systems. This formulation would reconcile the seemingly contradictory findings regarding the extent to which awareness is necessary to activate the amygdala. While not ruling out a necessary contribution from top-down structures, it raises the possibility that top-down contributions vary as a function of the stimuli ultimately perceived or suppressed from awareness.

Figure 6. Connectivity with the amygdala varies as a function of awareness. $\boldsymbol{a}$-c, Context-dependent connectivity analysis revealing areas with a significant modulation of functional connectivity with the right amygdala in the presence of perceived fearful trials relative to unperceived fearful trials $(p<0.01)$. Areas in orange and yellow show enhanced functional connectivity during perceived fearful trials relative to unperceived fearful trials: right superior temporal cortex $(\boldsymbol{a})$ and right middle temporal cortex $(\boldsymbol{b})$. Regions in blue show reduced functional connectivity with the right amygdala during perceived fearful trials relative to unperceived fearful trials: left perigenual prefrontal cortex (c).

(an effortful process), and the possible effects of these task demands on the current results remains unclear. Although future work that systematically varies cognitive demands will be needed to address this problem, our primary findings are not likely caused by a general emotion categorization difficulty effect. For example, other studies show that frontoparietal activity increases with heightened demands on attention, decision-making, or rule-learning (Mitchell et al., 2007, 2009; Dumontheil et al., 2010). However, we observed greatest frontoparietal activity to neutral faces, which our behavioral results (reaction time and error data) suggest were the easiest to classify in the absence of emotional competition.

\section{Emotion as a bottom-up influence on conscious perception}

It has been suggested that emotion acts as a bottom-up influence on stimulus representation, giving affective stimuli enhanced access to processing resources (Pessoa and Ungerleider, 2004; Blair and Mitchell, 2009). This influence is not sufficient to produce awareness; robust amygdala activity has been observed even when emotional awareness is reduced because of masking (Whalen et al., 1998; Morris et al., 1999) or cortical blindness (Morris et al., 2001; Pegna et al., 2005). One possibility is that the amygdala's response to fear is entirely independent of awareness. However, in the current study fearful faces were significantly

\section{Frontoparietal contributions to consciousness vary as a function of emotion}

Our results shed light on how top-down and bottom-up areas interact in some circumstances to influence consciousness. Frontoparietal networks are thought to make a generic (i.e., stimulus-independent) contribution to conscious perception (Lumer et al., 1998; Beck et al., 2001). We observed greater activity in dorsolateral prefrontal cortex (BA 8/9) and, to a lesser degree, inferior parietal cortex (BA 40/7) to perceived neutral relative to fearful or disgusted expressions. Despite being primed for awareness, fearful faces were associated with less activity in neural regions thought to play a critical role in consciousness (and greater amygdala activity). One interpretation of this finding is that the amygdala acts as a bottom-up amplifier that increases accessibility of emotional items to consciousness. Accordingly, because neutral stimuli would not benefit from similar bottom-up support, enhanced generic frontoparietal contributions become more important. This interpretation is consistent with proposals that frontoparietal regions contribute to consciousness by amplifying relevant sensory processes (Dehaene and Naccache, 2001; Sergent and Dehaene, 2004). It also raises the possibility that the amygdala makes a similar (though not sufficient) contribution to emotional awareness. However, it should be noted that stronger visual cortical responses have been observed for perceived compared with unperceived stimuli (Moutoussis and Zeki, 2002). Thus, it remains possible that the observed amygdala activity and enhanced functional coupling with the ventral visual system was the result of fear awareness rather than its cause. 


\section{Implications for emotion regulation}

The current results have implications for our understanding of emotion regulation. One proposed mechanism for emotion regulation involves projections between medial prefrontal cortex ( $\mathrm{mPFC}$ ) and basolateral amygdala. The $\mathrm{mPFC}$ is thought to regulate affect by modulating emotional output from the amygdala (Morgan et al., 1993; Rosenkranz and Grace, 2001). Furthermore, enhanced activity in $\mathrm{mPFC}$ is associated with reduced emotional distracter processing (Bishop et al., 2004) and successful emotional reappraisal (Ochsner et al., 2004). However, little is known about how activation of this system may vary as a function of awareness. In the present study, we found that activity in pgPFC was enhanced during the conscious perception of neutral relative to emotional facial expressions. Furthermore, the suppression of fearful stimuli from consciousness was associated with enhanced coupling between pgPFC and amygdala. These findings raise the intriguing possibility that activity in pgPFC may influence not only how one feels about a particular stimulus but also whether or not an emotional stimulus is accessible to awareness. An alternative possibility is that information about the presence of fearful stimuli is conveyed from amygdala to pgPFC, and it is in the latter structure that competition for emotional awareness is ultimately resolved.

\section{Relationship to other binocular rivalry studies of emotion} It is important to note that the current study differs in several key ways from prior binocular rivalry studies suggesting that amygdala activity is resistant to manipulations of awareness (Anderson et al., 2003; Williams et al., 2004). First, in these studies awareness of emotion was not the target process; emotional representation occurred incidentally while participants made other perceptual judgments (e.g., gender discriminations). The focus of these studies, therefore, was on emotional representation as a function of awareness rather than awareness of emotion per se. To our knowledge, this is the only fMRI study of binocular rivalry that targets awareness of emotionality within the same class of stimuli. Furthermore, whereas the current study involved competition between two faces for awareness, prior studies involved rivalry between faces and places. The use of two social stimuli that activate similar areas within the ventral stream may have enhanced competition. Indeed, we have noted previously that competition between discordant emotional expressions modulates the BOLD response in the ventral stream (Amting et al., 2009). It should be noted that by using face-only rivalry in the current study, we may have unintentionally increased perceptual fusion of the compound stimuli, with only piecemeal rivalry in parts of the face with the greatest discrepancy. The potential implications of this for our results remain unclear.

\section{Conclusions}

Considerable research supports the idea that contributions from the ventral visual system to consciousness vary in a categoryselective manner. However, to date little evidence exists that contributions from frontoparietal areas considered critical for consciousness are also variable. Here, we show that activity in these regions can vary as a function of the emotion perceived and suppressed from awareness. Strikingly, although fearful stimuli were more likely to be perceived when present, this increased awareness was associated with reduced activity in regions of frontoparietal cortices thought to play a critical and general role in consciousness. These results are consistent with the idea that the amygdala modulates the threshold for awareness of emotional stimuli. Evidence is also provided that activity in regions of pg-
PFC, previously implicated in extinguishing a fear-conditioned response and in explicit emotion regulation, is associated with the suppression of fearful faces from consciousness. The results have implications for models of consciousness, emotion regulation, and the relationship between amygdala activation and visual awareness.

\section{References}

Adolphs R, Tranel D, Damasio H, Damasio A (1994) Impaired recognition of emotion in facial expressions following bilateral damage to the human amygdala. Nature 372:669-672.

Amting JM, Miller J, Chow M, Mitchell DGV (2009) Getting mixed messages: the impact of conflicting social signals on the brain's target emotional response. Neuroimage 47:1950-1959.

Anderson AK, Phelps EA (2001) Lesions of the human amygdala impair enhanced perception of emotionally salient events. Nature 411:305-309.

Anderson AK, Christoff K, Panitz D, De Rosa E, Gabrieli JD (2003) Neural correlates of the automatic processing of threat facial signals. J Neurosci 23:5627-5633.

Bar M, Tootell RB, Schacter DL, Greve DN, Fischl B, Mendola JD, Rosen BR, Dale AM (2001) Cortical mechanisms specific to explicit visual object recognition. Neuron 29:529-535.

Beck DM, Rees G, Frith CD, Lavie N (2001) Neural correlates of change detection and change blindness. Nat Neurosci 4:645-650.

Bishop SJ, Duncan J, Lawrence AD (2004) State anxiety modulation of the amygdala response to unattended threat-related stimuli. J Neurosci 24:10364-10368.

Blair RJR, Mitchell DGV (2009) Psychopathy, attention and emotion. Psychol Med 39:543-555.

Calder AJ, Lawrence AD, Young AW (2001) Neuropsychology of fear and loathing. Nat Rev Neurosci 2:352-363.

Cox RW (1996) AFNI: software for analysis and visualization of functional magnetic resonance images. Comput Biomed Res 29:162-173.

Crick F, Koch C (2003) A framework for consciousness. Nat Neurosci 6:119-126.

de Gelder B, Vroomen J, Pourtois G, Weiskrantz L (1999) Non-conscious recognition of affect in the absence of striate cortex. Neuroreport 10:3759-3763.

de Gelder B, Morris JS, Dolan RJ (2005) Unconscious fear influences emotional awareness of faces and voices. Proc Natl Acad Sci U SA 102:18682-18687.

Dehaene S, Naccache L (2001) Towards a cognitive neuroscience of consciousness: basic evidence and a workspace framework. Cognition 79:1-37.

Dehaene S, Naccache L, Le Clec HG, Koechlin E, Mueller M, DehaeneLambertz G, van de Moortele PF, Le Bihan D (1998) Imaging unconscious semantic priming. Nature 395:597-600.

Dehaene S, Changeux JP, Naccache L, Sackur J, Sergent C (2006) Conscious, preconscious, and subliminal processing: a testable taxonomy. Trends Cogn Sci 10:204-211.

Del Cul A, Dehaene S, Reyes P, Bravo E, Slachevsky A (2009) Causal role of prefrontal cortex in the threshold for access to consciousness. Brain 132:2531-2540.

Desimone R, Duncan J (1995) Neural mechanisms of selective visual attention. Annu Rev Neurosci 18:193-222.

Driver J, Mattingley JB (1998) Parietal neglect and visual awareness. Nat Neurosci 1:17-22.

Dumontheil I, Thompson R, Duncan J (2010) Assembly and use of new task rules in fronto-parietal cortex. J Cogn Neurosci. Advance online publication. Retrieved June 28, 2010. doi:10.1162/jocn.2010.21438.

Ekman P, Friesen WV (1975) Unmasking the face: a guide to recognizing emotions from facial cues. Englewood Cliffs, New Jersey: Prentice-Hall.

Grill-Spector K, Kushnir T, Hendler T, Malach R (2000) The dynamics of object-selective activation correlate with recognition performance in humans. Nat Neurosci 3:837-843.

LeDoux J (1996) The emotional brain. New York: Simon and Schuster.

Leopold DA, Logothetis NK (1996) Activity changes in early visual cortex reflect monkeys' percepts during binocular rivalry. Nature 379:549-553.

Lumer ED, Friston KJ, Rees G (1998) Neural correlates of perceptual rivalry in the human brain. Science 280:1930-1934.

Lundqvist D, Flykt A, Ohman A (1998) The Karolinska Directed Emotional Faces (KDEF), CD-ROM from Department of Clinical Neuroscience, Psychology Section, Karolinska Institutet, ISBN 91-630-7164-9. 
Mitchell DGV, Nakic M, Fridberg D, Kamel N, Pine DS, Blair RJR (2007) The impact of processing load on emotion. Neuroimage 34:1299-1309.

Mitchell DGV, Luo Q, Avny SB, Kasprzycki T, Gupta K, Chen G, Finger EC, Blair RJR (2009) Adapting to dynamic stimulus-response values: differential contributions of inferior frontal, dorsomedial, and dorsolateral regions of prefrontal cortex to decision making. J Neurosci 29: 10827-10834.

Morgan MA, Romanski LM, LeDoux JE (1993) Extinction of emotional learning: contribution of medial prefrontal cortex. Neurosci Lett 163:109-113.

Morris JS, Ohman A, Dolan RJ (1999) A subcortical pathway to the right amygdala mediating "unseen" fear. Proc Natl Acad Sci USA 96: 1680-1685.

Morris JS, DeGelder B, Weiskrantz L, Dolan RJ (2001) Differential extrageniculostriate and amygdala responses to presentation of emotional faces in a cortically blind field. Brain 124:1241-1252.

Moutoussis K, Zeki S (2002) The relationship between cortical activation and perception investigated with invisible stimuli. Proc Natl Acad Sci U S A 99:9527-9532.

Ochsner KN, Gross JJ (2005) The cognitive control of emotion. Trends Cogn Sci 9:242-249.

Ochsner KN, Ray RD, Cooper JC, Robertson ER, Chopra S, Gabrieli JD, Gross JJ (2004) For better or for worse: neural systems supporting the cognitive down- and up-regulation of negative emotion. Neuroimage 23:483-499.

Pearson J, Brascamp J (2008) Sensory memory for ambiguous vision. Trends Cogn Sci 12:334-341.

Pegna AJ, Khateb A, Lazeyras F, Seghier ML (2005) Discriminating emotional faces without primary visual cortices involves the right amygdala. Nat Neurosci 8:24-25.

Pessoa L, Ungerleider LG (2004) Neuroimaging studies of attention and the processing of emotion-laden stimuli. Prog Brain Res 144:171-182.

Pessoa L, Japee S, Sturman D, Ungerleider LG (2006) Target visibility and visual awareness modulate amygdala responses to fearful faces. Cereb Cortex 16:366-375.

Quirk GJ, Gehlert DR (2003) Inhibition of the amygdala: key to pathological states? Ann NY Acad Sci 985:263-272.

Rees G (2007) Neural correlates of the contents of visual awareness in humans. Philos Trans R Soc Lond B Biol Sci 362:877-886.

Rosenkranz JA, Grace AA (2001) Dopamine attenuates prefrontal cortical suppression of sensory inputs to the basolateral amygdala of rats. J Neurosci 21:4090-4103.

Schneider W, Eschman A, Zuccolotto A (2002) E-Prime user's guide. Pittsburgh: Psychology Software Tools.

Sergent C, Dehaene S (2004) Neural processes underlying conscious per- ception: experimental findings and a global neuronal workspace framework. J Physiol (Paris) 98:374-384.

Sergerie K, Chochol C, Armony JL (2008) The role of the amygdala in emotional processing: a quantitative meta-analysis of functional neuroimaging studies. Neurosci Biobehav Rev 32:811-830.

Sterzer P, Rees G (2008) A neural basis for percept stabilization in binocular rivalry. J Cogn Neurosci 20:389-399.

Tamietto M, Castelli L, Vighetti S, Perozzo P, Geminiani G, Weiskrantz L, de Gelder B (2009) Unseen facial and bodily expressions trigger fast emotional reactions. Proc Natl Acad Sci U S A 106:17661-17666.

Tong F, Nakayama K, Vaughan JT, Kanwisher N (1998) Binocular rivalry and visual awareness in human extrastriate cortex. Neuron 21:753-759.

Tong F, Meng M, Blake R (2006) Neural bases of binocular rivalry. Trends Cogn Sci 10:502-511.

Tottenham N, Borscheid A, Ellertsen K, Marcus DJ, Nelson CA (2002) Categorization of facial expressions in children and adults: establishing a larger stimulus set. J Cogn Neurosci [Suppl] 14:S74.

Tse PU, Martinez-Conde S, Schlegel AA, Macknik SL (2005) Visibility, visual awareness, and visual masking of simple unattended targets are confined to areas in the occipital cortex beyond human V1/V2. Proc Natl Acad Sci U S A 102:17178-17183.

Vuilleumier P, Driver J (2007) Modulation of visual processing by attention and emotion: windows on causal interactions between human brain regions. Philos Trans R Soc Lond B Biol Sci 362:837-855.

Vuilleumier P, Sagiv N, Hazeltine E, Poldrack RA, Swick D, Rafal RD, Gabrieli JD (2001a) Neural fate of seen and unseen faces in visuospatial neglect: a combined event-related functional MRI and event-related potential study. Proc Natl Acad Sci U S A 98:3495-3500.

Vuilleumier P, Armony JL, Driver J, Dolan RJ (2001b) Effects of attention and emotion on face processing in the human brain: an event-related fMRI study. Neuron 30:829-841.

Vuilleumier P, Armony JL, Clarke K, Husain M, Driver J, Dolan RJ (2002) Neural response to emotional faces with and without awareness: eventrelated fMRI in a parietal patient with visual extinction and spatial neglect. Neuropsychologia 40:2156-2166.

Whalen PJ, Rauch SL, Etcoff NL, McInerney SC, Lee MB, Jenike MA (1998) Masked presentations of emotional facial expressions modulate amygdala activity without explicit knowledge. J Neurosci 18:411-418.

Williams MA, Morris AP, McGlone F, Abbott DF, Mattingley JB (2004) Amygdala responses to fearful and happy facial expressions under conditions of binocular suppression. J Neurosci 24:2898-2904.

Wright CI, Fischer H, Whalen PJ, McInerney SC, Shin LM, Rauch SL (2001) Differential prefrontal cortex and amygdala habituation to repeatedly presented emotional stimuli. Neuroreport 12:379-383. 